\title{
Apnea Testing During Brain Death Assessment: A Review of Clinical Practice and Published Literature
}

\author{
J Brady Scott RRT-ACCS, Michael A Gentile RRT FAARC, Stacey N Bennett MSN ACNP, \\ MaryAnn Couture RRT, and Neil R MacIntyre MD FAARC
}

\begin{abstract}
The diagnosis of brain death is a complex process. Strong knowledge of neurophysiology and an understanding of brain death etiology must be used to confidently determine brain death. The key findings in brain death are unresponsiveness, and absence of brainstem reflexes in the setting of a devastating neurological injury. These findings are coupled with a series of confirmatory tests, and the diagnosis of brain death is established based on consensus recommendations. The drive to breathe in the setting of an intense ventilatory stimulus (ie, respiratory acidosis) is a critical marker of brainstem function. As a consequence, apnea testing is an important component of brain death assessment. This procedure requires close monitoring of a patient as all ventilator support is temporarily removed and $\mathrm{PaCO}_{2}$ levels are allowed to rise. A "positive" test is defined by a total absence of respiratory efforts under these conditions. While apnea testing is not new, it still lacks consensus standardization regarding the actual procedure, monitored parameters, and evidencebased safety measures that may be used to prevent complications. The purpose of this report is to provide an overview of apnea testing and discuss issues related to the administration and safety of the procedure. Key words: apnea testing; brain death assessment; ventilatory drive assessment; transcutaneous carbon dioxide. [Respir Care 2013;58(3):532-538. (C) 2013 Daedalus Enterprises]
\end{abstract}

\section{Introduction}

The concept of brain death was first described in the medical literature in 1959, and was an attempt to separate the cessation of meaningful neurological function from the cessation of cardiorespiratory function. ${ }^{1}$ The rationale for this distinction was that the former represented human existence, while the latter was viewed simply as biological existence. However, despite more than half a century of the general acceptance of brain death as a medical concept, clear consensus regarding brain death determination is still

At the time of this research, Mr Scott was affiliated with the Department of Respiratory Care Services, Duke University Medical Center, Durham, North Carolina. He is now affiliated the Department of Respiratory Care, Rush University Medical Center, Chicago, Illinois. Mr Gentile and Dr MacIntyre are affiliated with the Division of Pulmonary and Critical Care Medicine, and Ms Bennett is affiliated with Neuroscience Critical Care, Duke University Medical Center, Durham, North Carolina. Ms Couture is affiliated with the Department of Respiratory Therapy, Hartford Hospital, Hartford, Connecticut.

The authors have disclosed no conflicts of interest. lacking. ${ }^{2}$ In general, the declaration of brain death usually requires that the cause of brain injury is known, the irreversibility of the injury is certain, and important neurological signs of brain function are absent.

The recognition of confounding factors, proper interpretation of appropriate neurologic imaging, and performance of any confirmatory examination and testing should be considered when determining brain death. Medical conditions possibly complicating the clinical assessments include severe acidosis/alkalemia, body temperature irregularities, drug intoxication, poisoning, neuromuscular blockade, and a locked-in syndrome. These circumstances must be considered before brain death determination can proceed. It is also important to understand some neurologic states can mimic brain death. ${ }^{3}$

Correspondence: J Brady Scott RRT-ACCS, Respiratory Care Services, Rush University Medical Center, 1620 West Harrison Street, LL 1202.13, Chicago IL 60612. E-mail: Jonathan_B_Scott@rush.edu.

DOI: $10.4187 /$ respcare. 01962 


\section{Brain Death Physiology}

Brain death was defined in 1995 by the American Academy of Neurology as the irreversible cessation of function of the brain, including the brainstem. ${ }^{4}$ Two clinical findings are necessary to confirm this irreparable condition of the brain: coma (with a known cause), and absence of brainstem reflexes. The cause of brain death itself is variable, and can be a result of traumatic events or numerous medical conditions. Brain death may be frequently seen as a consequence of aneurysmal subarachnoid hemorrhage, intracerebral hemorrhage or infarct, cardiac arrest, and severe head injury. Regardless of the primary insult to the brain, tissue death occurs from brain anoxia by one of several mechanisms: severe blood hypoxemia (ie, lethal reductions in nutrient or oxygen blood content); severely impaired perfusion (eg, widespread edema, elevation of intracranial pressure; the cessation of blood flow to the brain and brainstem); or toxic neuronal injury.

Cerebral blood flow is particularly important when considering a diagnosis of brain death. Reduction of blood flow to the brain is usually created when the intracranial pressure is greater than the mean arterial pressure, thus lowering the cerebral perfusion pressure to a point that sufficient blood flow into the brain ceases. This lack of blood flow to the brain usually results in widespread cell anoxia and death. While this physiologic mechanism is considered the most devastating, it is not the only one described. Another pattern is characterized by intact cerebral blood flow, which should adequately deliver oxygen, glucose, and other nutrients to the brain at sufficient levels to sustain tissue life. However, in this pattern an intrinsic catastrophic event occurs at the cellular level, which prevents extraction or utilization of the oxygen and nutrients needed to maintain cellular function and metabolism. Examples include cyanide poisoning and other metabolic derangements. This mechanism results in lack of oxygenation to the brain, not by failure of the delivery system, but from end organ breakdown of the brain, at the capillary or tissue level. ${ }^{5}$

\section{Ethical and Legal Issues}

Historically, brain death and cardiorespiratory death often occurred together. However, the use of mechanical ventilation to prevent respiratory arrest, in conjunction with the improvement of blood pressure management, has transformed the course of terminal neurological disorders. Cardiorespiratory functions can now be mechanically maintained after the brain has ceased to function. This has led to ethical debates about the definition of brain death and the concept of human death. The American Academy of Neurology's definition of brain death is considered the accepted medical definition. However, it is important to
Table 1. Brain Death Guidelines

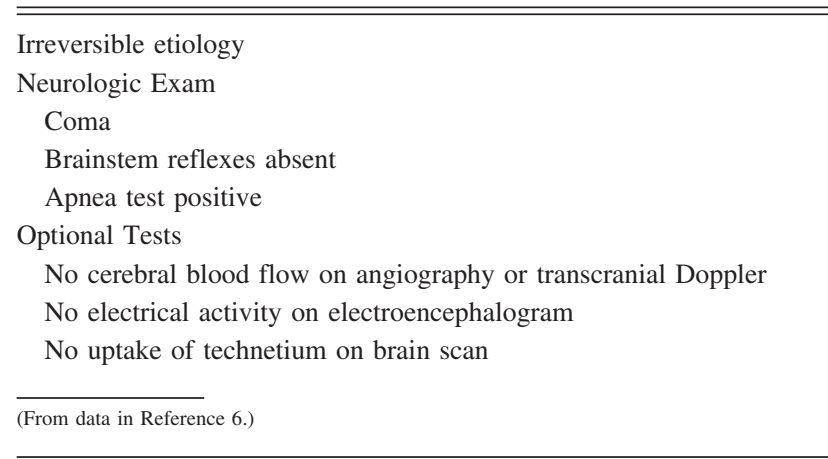

understand that brain death essentially has 2 definitions: one is medical and the other is legal. The legal definition is set by the individual states, which are responsible for defining "death" for legal reasons.

Beyond medical definitions and considerations, cultural and regional acceptance of death varies and must be addressed. For example, some cultures and religions do not accept death until after all vital functions of the body have ceased. ${ }^{1}$ Many, regardless of culture or religion, may find it very difficult to accept death when the heart and lungs appear to still be living, although being completely supported by mechanical and pharmacologic means. However, when brain death is determined and documented, many states no longer require the continuation of cardiorespiratory life support. The decision to withdraw cardiorespiratory life support based on the concept of brain death may be difficult for many caregivers and family members. The clinical staff is responsible for clearly defining and communicating the process to those involved in patient decision making.

Individual hospitals are typically responsible for creating written policies to clearly specify the process of determining brain death. These may include a description of the examinations and diagnostic tests, and which individuals should be present to make the conclusion. Published guidelines for establishing brain death are available (Table 1). ${ }^{6}$ The evidence supporting these guidelines is not robust, and it is noted that some data supporting these recommendations are inadequate or conflicting. ${ }^{6}$

\section{Determining Brain Death: The Role of Apnea Testing}

Apnea testing is an essential component in the clinical determination of brain death. ${ }^{6,7}$ The main objective of apnea testing is to prove the absence of respiratory control system reflexes in the brainstem when intense physiologic stimulation to breathe takes place. In the case of apnea testing, the stimulus is the rapid increase in the $\mathrm{PaCO}_{2}$ to $>60 \mathrm{~mm} \mathrm{Hg}$ (or a $20 \mathrm{~mm} \mathrm{Hg}$ increase in $\mathrm{PaCO}_{2}$ over 
Table 2. Survey of Clinical Practice of Apnea Brain Death Assessment

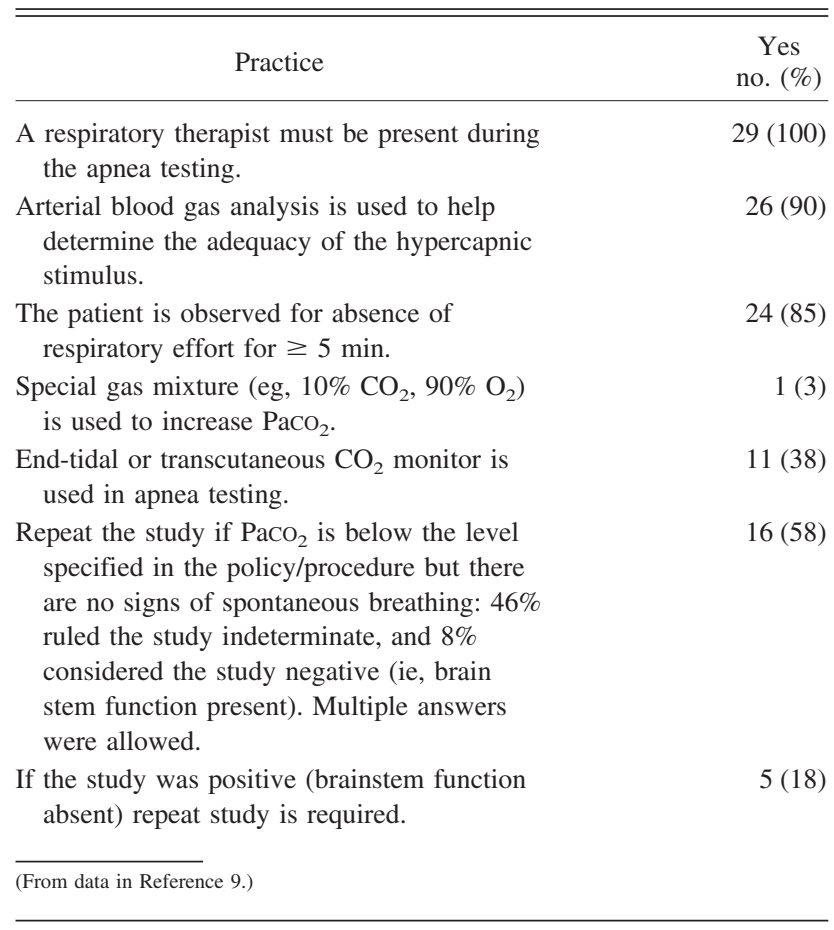

baseline normal $\mathrm{PaCO}_{2}$ ), and the corresponding decrease in $\mathrm{pH}$ before buffering mechanisms can occur. ${ }^{6,8}$

Because of the potential implications involved in the diagnosis of brain death, it is essential that apnea testing be safe, accurate, and reproducible. This not only serves the clinicians who are tasked with diagnosing brain death, but also allows family members of patients to rest more assured that the decisions regarding end of life issues were made with sound, informed, and proper judgment. The outcome of an apnea testing is very straightforward. A negative test is defined by any spontaneous respiratory efforts in response to hypercapnic/acidotic stimulation; a positive test is the absence of any respiratory activity under these conditions.

Wide practice variability exists in regard to apnea testing. A survey of respiratory therapists illustrated some of these variations, revealing disparities in both monitoring and methods (Table 2). ${ }^{9}$ There are potentially several reasons for different standards. Apnea testing is a low volume procedure. For example, large tertiary care hospitals may diagnose brain death only 25-30 times per year. ${ }^{10}$ The only universal agreement among surveyed participants was that respiratory therapists were to be present during all apnea tests. Recently, the apnea testing itself and its utility have been questioned. ${ }^{11,12}$ Also, substantial discussion among organ procurement organizations and clinicians has been provoked, due to the suggestion that only one clinical exam, along with the apnea test, may be enough to ade- quately and reasonably diagnose brain death. This further emphasizes the need for well designed studies involving the safety, efficiency, and effectiveness of the various methods of apnea testing.

\section{Complications of Apnea Testing}

In order to be considered a useful tool in the determination of brain death, apnea testing must be rigorous enough to reliably demonstrate apnea and brainstem death without causing further organ damage. ${ }^{13}$ Thus, care must be taken to avoid iatrogenic injury during apnea testing. Complications associated with apnea testing include hypoxemia, hypotension, acidosis, hypercapnia, increased intracranial pressure, pulmonary hypertension, and cardiac arrhythmias (Table 3). ${ }^{14-20}$ Cases of cardiac arrest and pneumothorax have also been reported..$^{21}$

Certain complications can be considered inherent, due to the nature of the test itself. For example, some degree of respiratory acidosis and hypercapnia will be present if the patient is indeed apneic and carbon dioxide production in the body continues. While this is an expected response, the clinicians performing the study must be aware of the potential for severe acidemia and possible deleterious side effects. Carbon dioxide and acidemia cause peripheral vasodilation and cardiac depression, frequently resulting in hypotension, which is one of the most common complications of apnea testing. ${ }^{17,22,23}$ The impaired autonomic reflexes found in many of these patients increase the potential for blood pressure problems. Hypoxemia during apnea testing further increases the potential risk for cardiac dysrhythmias and hypotension. Appropriate precautionary steps, such as pre-oxygenation with 100\% oxygen, are essential to prevent hypoxia during apnea testing, should be used, and have been shown to be effective. ${ }^{14,17}$

Other very serious complications of the apnea test have also been noted. Burns and Russell reported a case in which their patient developed a tension pneumothorax, pneumomediastinum, and pneumoperitoneum as a result of airway perforation from the oxygen catheter. ${ }^{24}$ BarJoseph et al also reported 2 cases of tension pneumothorax that they concluded were the result of massive air trapping beyond a cannula that was obstructing the airway. ${ }^{25}$ In the cases presented, both patients subsequently deteriorated rather quickly: one patient expired immediately after a cardiopulmonary resuscitation attempt; the other expired shortly after the condition was stabilized and was confirmed brain dead one hour later. Additionally, in the latter case the heart and lungs were damaged severely and were unable to be used for transplantation. The authors recommended use of a markedly smaller cannula than the inner diameter of the endotracheal tube during the apneic oxygenation procedure, to prevent air trapping. They con- 
Apnea Testing During Brain Death Assessment

Table 3. Apnea Test Studies

\begin{tabular}{|c|c|c|c|c|c|c|c|c|c|}
\hline $\begin{array}{l}\text { First } \\
\text { Author }\end{array}$ & Year & $n$ & Study Design & $\begin{array}{c}\text { Cardiac } \\
\text { Arrest } \\
\text { no. }(\%)\end{array}$ & $\begin{array}{l}\text { Low } \\
\text { Blood } \\
\text { Pressure } \\
\text { no. }(\%)\end{array}$ & $\begin{array}{l}\text { Low } \\
\text { Heart } \\
\text { Rate } \\
\text { no. }(\%)\end{array}$ & $\begin{array}{l}\text { Arrhythmia } \\
\text { no. }(\%)\end{array}$ & $\begin{array}{l}\text { Hypoxemia } \\
\text { no. }(\%)\end{array}$ & Protocol/Principal Findings \\
\hline Benzel $^{14}$ & 1992 & 11 & Retrospective & & & & & & $\begin{array}{l}\mathrm{O}_{2} \text { flow not described. } \\
\text { Determined } \mathrm{CO}_{2} \text { rise was } \\
5.1 \mathrm{~mm} \mathrm{Hg} / \mathrm{min} \\
\text { No complications }\end{array}$ \\
\hline Jeret $^{15}$ & 1994 & 61 & Retrospective & & $23(33)$ & & & $3(4)$ & $\begin{array}{l}\text { Up to } 10 \text { min per tests } \\
\text { Inconsistent arterial blood draws up } \\
\text { to } 23 \text { patients }\end{array}$ \\
\hline Schafer ${ }^{16}$ & 1978 & 10 & Non-randomized & & $1(10)$ & & $1(10)$ & & $\begin{array}{l}\text { Described procedure with } 6 \mathrm{~L} / \mathrm{min} \\
\mathrm{O}_{2} \\
\text { Determined } \mathrm{CO}_{2} \text { rise was } \\
\quad>3.2 \mathrm{~mm} \mathrm{Hg} / \mathrm{min}\end{array}$ \\
\hline Goudreau $^{17}$ & 2000 & 121 & Retrospective & $1(<1)$ & $34(28)$ & & $2(2)$ & & $\begin{array}{l}\text { Pre-oxygenation was described only } \\
\text { with ventilator disconnect }\end{array}$ \\
\hline Melano $^{8}$ & 2002 & 200 & Retrospective, non-randomized & $1(1)$ & $8(12)$ & & $1(1)$ & $17(25)$ & $\begin{array}{l}\text { Subjects randomized and compared } \\
\text { to alternative methods }\end{array}$ \\
\hline Saposnik $^{18}$ & 2004 & 129 & Retrospective & $2(3)$ & $13(20)$ & $2(3)$ & $2(3)$ & $4(6)$ & $\begin{array}{l}\text { Defined parameters for severe } \\
\text { acidosis, hypoxemia, systolic and } \\
\text { mean blood pressure } \\
\text { complications }\end{array}$ \\
\hline Lévesque $^{19}$ & 2006 & 20 & Repeated measures & & & & $1(5)$ & $2(10)$ & $\begin{array}{l}\text { American Academy of Neurology } \\
\text { guidelines compared to } \\
\text { alternative test procedures }\end{array}$ \\
\hline Vivien $^{13}$ & 2006 & 32 & Retrospective, non-randomized & & $14(43)$ & & & & $\begin{array}{l}\text { Tests with } 9 \mathrm{~L} / \mathrm{min}_{2} \text { in the } \\
\text { endotracheal tube } \\
20 \text { patients had } 20 \text { min test } \\
12 \text { patients had transcutaneous } \\
\text { monitoring } \\
\text { Assessed transcutaneous } \mathrm{CO}_{2} \\
\text { monitoring, which met goals } \\
\text { within } 11 \text { min }\end{array}$ \\
\hline Wijdicks ${ }^{20}$ & 2008 & $\begin{array}{c}195 \text { adult } \\
33 \text { pediatric } \\
212 \text { total tests }\end{array}$ & Retrospective & & $14(7)$ & & & $10(5)$ & $\begin{array}{l}\mathrm{O}_{2} \text { delivered at } 6-10 \mathrm{~L} / \mathrm{min} \text { inserted } \\
\text { in endotracheal tube } \\
\text { A T-piece with CPAP variation was } \\
\text { used on } 1 \text { patient. } \\
3 \text { patients had an anesthesia bag } \\
\text { attached to the endotracheal tube. } \\
7 \text { tests were aborted. }\end{array}$ \\
\hline $\begin{array}{l}\text { Totals, no. } \\
\quad(\%)\end{array}$ & & $608(100)$ & & $4(<1)$ & $111(18)$ & $2(<1)$ & $7(1)$ & $36(6)$ & \\
\hline
\end{tabular}

cluded with a recommendation that all guidelines for the determination of brain death contain detailed instructions regarding the safe performance of apnea testing, ensuring adequate oxygenation and the prevention of inadvertent pneumothorax.

\section{Methods of Performing Apnea Testing}

Various methods to carry out apnea testing procedures vary from institution to institution, yet a core set of procedural elements are common (Table 4). Immediately before all apnea tests, an initial arterial blood gas sample should be obtained to establish baseline $\mathrm{PaCO}_{2}$ levels. This information will be useful when making the final determination of whether or not the increase in arterial carbon dioxide/corresponding decrease in $\mathrm{pH}$ have occurred.

A common method of apnea testing involves disconnection of mechanical ventilation from the patient, followed by the insertion of a catheter or cannula into the endotracheal tube, down to the level of the carina, through 
Table 4. Apnea Testing Guidelines

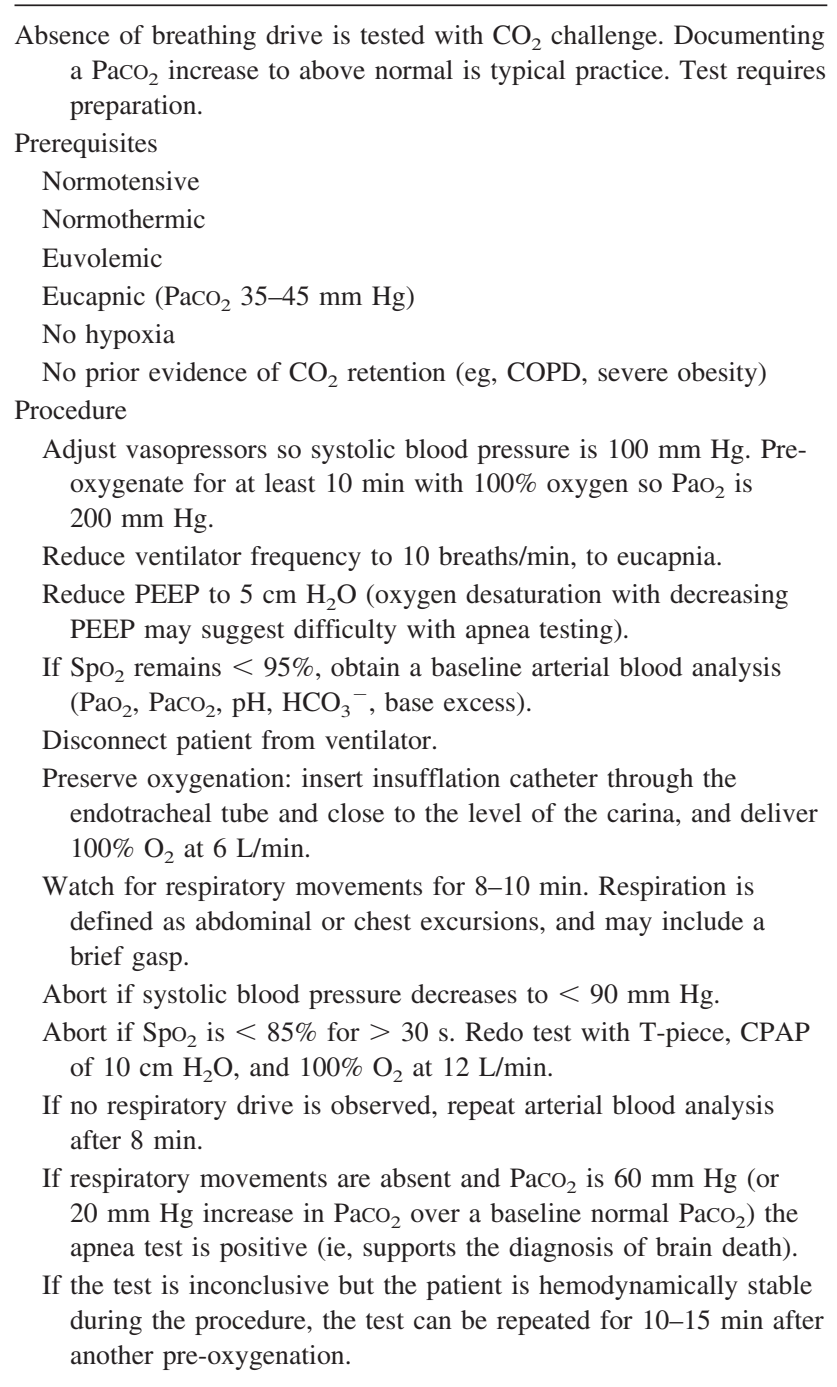

(From data in Reference 6.)

which oxygen is delivered. ${ }^{4,10,26}$ Disconnection from mechanical ventilation is thought to prevent erroneous ventilation readings by the ventilator itself. This is termed the apneic oxygenation method. Oxygen flow is usually 4$10 \mathrm{~L} / \mathrm{min} .{ }^{6}$ This flow ensures adequate delivery of oxygen to the blood through gas displacement in the upper bronchial tree, and diffusion to the alveoli, even in the absence of respiratory movement.

The duration of time of mechanical ventilation disconnection needs to be sufficient to raise the $\mathrm{PaCO}_{2}$ to $>60 \mathrm{~mm} \mathrm{Hg}$. Current guidelines for apnea testing state that measurement of the blood gas should be conducted after approximately 8-10 min. ${ }^{6}$ However, relying on fixed durations, which are based upon the expected rise in $\mathrm{PaCO}_{2}$ with apnea (3-4 $\mathrm{mm} \mathrm{Hg} / \mathrm{min}$ under normal physiologic conditions), may yield variable results, as the relationship of $\mathrm{PaCO}_{2}$ and $\mathrm{CO}_{2}$ production may vary greatly under conditions of terminal illness and brain death. ${ }^{27}$ Because of this, the apnea testing may be continued until the arterial blood gas results are noted, as long as the patient remains hemodynamically stable and with an adequate $\mathrm{Spo}_{2}$. Continuing the apnea test for slightly longer periods (eg, up to $15 \mathrm{~min}$ ) may be preferable to repeating the test. Additionally, $\mathrm{CO}_{2}$ elevation and elimination may be skewed by the oxygen flow via a catheter placed in the endotracheal tube. There are modifications of the apneic oxygenation method that serve to refine and streamline apnea testing. Benzel et al modified their protocol by initially reducing mechanical ventilation settings to allow for a higher initial $\mathrm{PaCO}_{2}$ at baseline. This was shown to reduce the amount of time required to reach the desired goal of $60 \mathrm{~mm} \mathrm{Hg} .{ }^{14}$ As in other studies, they also noted that the rate of $\mathrm{PaCO}_{2}$ elevation varies considerably from patient to patient, regardless of baseline $\mathrm{PaCO}_{2}$.

Different technical variations of the apneic oxygenation technique for apnea testing have also been described. Tpiece systems used to provide oxygen via standard corrugated tubing connected to $100 \%$ oxygen at a flow of $12 \mathrm{~L} /$ min have been shown to be as effective as the previously described oxygen cannula technique. ${ }^{19}$ This method of apnea testing may theoretically be safer than the intratracheal catheter method, since the possible risk of increased intrathoracic pressure or pneumothorax would be lower. The investigators further modified their T-piece approach with corrugated tubing technique, with a $10 \mathrm{~cm} \mathrm{H}_{2} \mathrm{O}$ CPAP valve attached to the distal extremity of the T-piece extension to enhance oxygenation. ${ }^{19}$

Additional methods that allow the patient to stay connected to a mechanical ventilator circuit until the final phase of the apnea testing have been described. In one method, hypoventilation is achieved by reducing the minute ventilation to a low level and keeping the patient on the ventilator until the required $\mathrm{PaCO}_{2}$ is attained. At this point the ventilator is disconnected. The authors recommend the patient be observed during this disconnection period for any respiratory movements, suggesting that 30-60 seconds is an adequate amount of time to observe for apnea. ${ }^{14}$ A modification of this method allows the patient to remain connected to the ventilator circuit in order to provide an elevated CPAP while the ventilator supplies no breaths. ${ }^{28}$

Artificial augmentation of $\mathrm{PaCO}_{2}$ levels has also been considered an option for apnea testing. ${ }^{14,17,29,30}$ This method involves the exogenous administration of $\mathrm{CO}_{2}$ into the ventilator circuit to achieve the desired $\mathrm{PaCO}_{2}$ level during the apnea testing. Different mixtures of oxygen/carbon dioxide (eg, $10 \% \mathrm{CO}_{2}$, balance $\mathrm{O}_{2}$ ), sometimes referred to as carbogen, may be used to achieve these desired levels. The delivery of carbogen may be accomplished by utilizing a bag-valve system attached to the tank, or by attaching the tank to the ventilator itself. ${ }^{29,31}$ Other means of 
delivering $\mathrm{CO}_{2}$ have been described, such as the administration of pure $\mathrm{CO}_{2}$ into the inspiratory limb of the ventilator, without modifying other parameters such as the $\mathrm{FIO}_{2}{ }^{8}$ The obvious advantage of providing $\mathrm{CO}_{2}$ in the ventilator circuit is that the desired $\mathrm{PaCO}_{2}$ level can be reached very quickly, and thus the risks associated with hypoxemia are minimized. The procedure is generally monitored with an exhaled $\mathrm{CO}_{2}$ analyzer; when the desired level is reached (eg, a $20 \mathrm{~mm} \mathrm{Hg}$ increase), the ventilator is either disconnected or switched to CPAP and the patient is monitored for 1-2 min for respiratory efforts.

All of the above apnea testing procedures have a simple common goal: elevate $\mathrm{PaCO}_{2}$ and observe the patient for any spontaneous respiratory effort to reflect brainstem activity. It must be emphasized that spontaneous respiratory efforts may be subtle, so very close observation of the patient's chest and abdomen with an unobstructed view is essential. Further study to determine if one method is more safe, effective, and reproducible is warranted in order to establish guidelines to be used by both the expert and the novice clinician.

\section{Monitoring/Precautions During Apnea Testing}

Continuous patient monitoring during apnea testing is imperative. Due to the very nature of the clinical condition of the patient, apnea testing is most likely performed in a critical care area where monitoring and close patient supervision are provided. Physiologic parameters, including heart rate/rhythm, arterial blood pressure, exhaled $\mathrm{CO}_{2}$, and $\mathrm{SpO}_{2}$, should be continuously evaluated before, during, and after the apnea testing. Taking precautionary steps prior to apnea testing, such as ensuring normotension (eg, adequate intravenous access and intravenous vasopressor agents) and adequate pre-oxygenation, may be warranted to prevent complications. Goudreau et al found that cardiovascular complications were noted in approximately 1 in 4 apnea tests. ${ }^{17}$ This rate nearly doubled in tests performed in patients with unstable or borderline baseline blood pressure or $\mathrm{SpO}_{2}$. The authors emphasized that a majority ( $85 \%$ ) of apnea testing can be performed without complications when adequate precautions are taken. Clinicians need to be educated on the complexities of apnea testing, and efforts need to be made to improve on the proper execution of apnea testing to reduce the risk of complications.

Supplementary monitoring with transcutaneous $\mathrm{CO}_{2}$ monitoring during the apnea testing procedure may be considered (Fig. 1). Transcutaneous $\mathrm{CO}_{2}$ monitoring has been reported to prevent excessive hypercarbia/respiratory acidosis resulting from the apnea testing procedure. Vivien et al, in a cohort study evaluating the use of transcutaneous $\mathrm{CO}_{2}$ monitoring in brain death determination, found that it accurately predicts when the $\mathrm{PaCO}_{2}$ goal has been attained. ${ }^{13}$

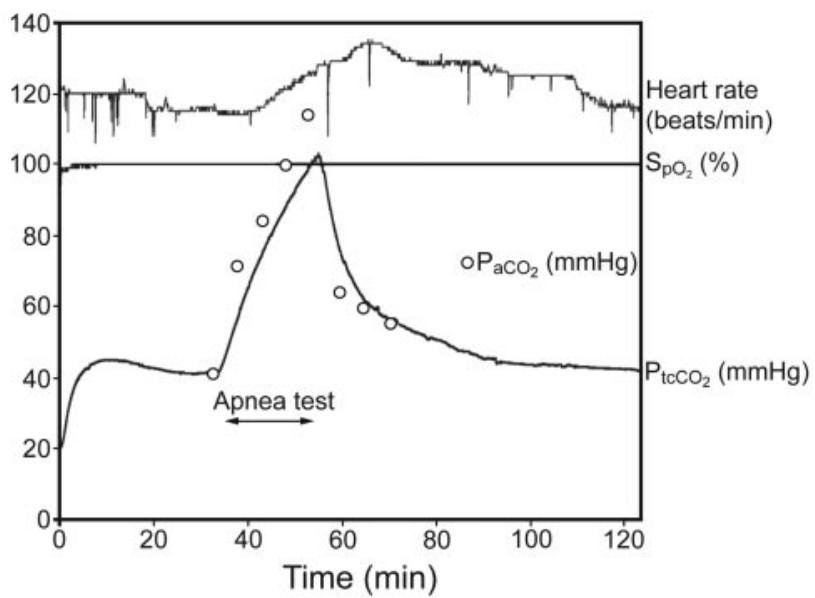

Fig. 1. Representative tracings of transcutaneous $\mathrm{CO}_{2}\left(\mathrm{P}_{\mathrm{tcCO}_{2}}\right)$, $\mathrm{SpO}_{2}$, and heart rate during an apnea test designed to raise $\mathrm{PaCO}_{2}$ to $100 \mathrm{~mm} \mathrm{Hg}$. At baseline, $\mathrm{P}_{\mathrm{tcCO}}$ is very close to $\mathrm{PacO}_{2}$, but exhibits a delay during the rapid increase of $\mathrm{PaCO}_{2}$ during apnea. After reconnection to the ventilator, $\mathrm{P}_{\mathrm{tcCO}}$ becomes closer to $\mathrm{PaCO}_{2}$, as the rate of decrease in $\mathrm{PaCO}_{2}$ was reduced. (From Reference 13 , with permission.)

Lang et al also reported a strong correlation between transcutaneous $\mathrm{CO}_{2}$ and $\mathrm{PaCO}_{2}$. They suggested this modality was effective at reducing $\mathrm{CO}_{2}$ target overshoot during exogenous $\mathrm{CO}_{2}$ administration. ${ }^{21}$ It is important to note that the $\mathrm{PaCO}_{2}$ sampling level, confirmed by arterial blood gas measurement, is the accepted standard.

\section{Summary}

Apnea testing is an integral component in brain death determination. Several attempts have been undertaken to better understand the best practice methods regarding the apnea testing. Most experts in the field of brain death determination consider apnea testing essential, but widely accepted guidelines and parameters, including detailed instructions regarding the procedure itself, do not yet exist. The safety and reliability of apnea testing are common themes when reviewing available literature. Debate continues regarding the specific parameters to be desired, met, and maintained. All known methods of apnea testing have yet to be fully scrutinized, although recommendations regarding their use have been published.

\section{REFERENCES}

1. Lazar NM, Shemie S, Webster GC, Dickens BM. Bioethics for clinicians. 24. Brain death. CMAJ 2001;164(6):833-836

2. Wijdicks EF. Brain death worldwide: accepted fact but no global consensus in diagnostic criteria. Neurology 2002;58(1):20-25.

3. Wijdicks EF. The diagnosis of brain death. N Engl J Med 2001; 344(16):1215-1221. 


\section{Apnea Testing During Brain Death Assessment}

4. The Quality Standards Subcommittee of the American Academy of Neurology. Practice parameters for determining brain death in adults. Neurology 1995;45(5):1012-1014.

5. Machado C. Diagnosis of brain death. Neurol Int 2010;2(1)e2.

6. Wijdicks EF, Varelas PN, Gronseth GS, Greer DM. Evidence-based guideline update: determining brain death in adults. Report of the Quality Standards Subcommittee of the American Academy of Neurology. Neurology 2010;74(23):1911-1918.

7. Sullivan J, Seem DL, Chabalewski F. Determining brain death. Crit Care Nurse 1999;19(2):37-46.

8. Melano R, Adum ME, Scarlatti A, Bazzano R, Araujo JL. Apnea test in the diagnosis of brain death: comparison of two methods and analysis of outcome. Transplant Proc 2002;34(1):11-12.

9. Scott JB, Gentile MA, Thalman JJ, MacIntyre NR. Survey on clinical practice of apnea brain death assessment (abstract). Respir Care 2008;53(11):1581.

10. Wijdicks EF. Determining brain death in adults. Neurology 1995; 45(5):1003-1011.

11. Joffe AR, Anton NR, Duff JP. The apnea test: rationale, confounders, and criticism. J Child Neurol 2010;25(11):1435-1443.

12. Tibballs J. A critique of the apneic oxygenation test for the diagnosis of "brain death". Pediatr Crit Care Med 2010;11(4):475-478.

13. Vivien B, Marmion F, Roche S, Devilliers C, Langeron O, Coriat P, et al. An evaluation of transcutaneous carbon dioxide partial pressure monitoring during apnea testing in brain-dead patients. Anesthesiology 2006;104(4):701-707.

14. Benzel EC, Mashburn JP, Conrad S, Modling D. Apnea testing for the determination of brain death: a modified protocol. J Neurosurg 1992;76(6):1029-1031.

15. Jeret JS, Benjamin JL. Risk of hypotension during apnea testing. Arch Neurol 1994;51(6):595-599.

16. Schafer JA, Caronna JJ. Duration of apnea needed to confirm brain death. Neurology 1978(7):28:661-666.

17. Goudreau JL, Wijdicks EF, Emery BA. Complications during apnea testing in the determination of brain death: predisposing factors. Neurology 2000;55(7):1045-1048.

18. Saposnik G, Rizzo G, Vega A, Sabbatiello R, Deluca JL. Problems associated with the apnea test in the diagnosis of brain death. Neurol India 2004;52(3):342-345.
19. Lévesque S, Lessard MR, Nicole PC, Langevin S, LeBlanc F, Lauzier $\mathrm{F}$, et al. Efficacy of a T-piece system and a continuous positive airway pressure system for apnea testing in the diagnosis of brain death. Crit Care Med 2006;34(8):2213-2216.

20. Wijdicks EF, Rabinstein AS, Manno EM, Atkinson JD. Pronouncing brain death: contemporary practice and safety of the apnea test. Neurology 2008;71(16):1240-1244.

21. Lang CJ. Apnea testing guided by continuous transcutaneous monitoring of partial pressure of carbon dioxide. Crit Care Med 1998; 26(5):868-872.

22. Wu WL, Fang Q, Li L, Qiu YQ, Luo BY. Complications associated with the apnea test in the determination of the brain death. Chin Med J 2008;121(13):169-1172.

23. Yee AH, Mandrekar J, Rabinstein AA, Wijdicks EF. Predictors of apnea test failure during brain death determination. Neurocrit Care 2010;12(3):352-355.

24. Burns JD, Russell JA. Tension pneumothorax complicating apnea testing during brain death evaluation. J Clin Neurosci 2008;15(5): 580-582.

25. Bar-Joseph G, Bar-Lavie Y, Zonis Z. Tension pneumothorax during apnea testing for the determination of brain death. Anesthesiology 1998;89(5):1250-1251.

26. Machado C. Brain death diagnosis. Acta Medica Medianae 2009; 48(3):25-30.

27. Machado C, Perez J, Scherle C, Areu A, Pando A. Brain death diagnosis and apnea test safety. Ann Indian Acad Neurol 2009;12(3): 197-200.

28. al Jumah M, McLean DR, al Rajeh S, Crow N. Bulk diffusion apnea test in the diagnosis of brain death. Crit Care Med 1992; 20(11):1564-1567.

29. Sharpe MD, Young GB, Harris C. The apnea test for brain death determination: an alternative approach. Neurocrit Care 2004;1(3): 363-366.

30. Lang CJ. Apnea testing by artificial $\mathrm{CO}_{2}$ augmentation. Neurology 1995;45(5):966-969.

31. Couture M, Pylant RT, Fancella CA. An alternative apnea test to determine brain death: capnography with carbogen administration (abstract). Respir Care 2007;52(11):1567. 